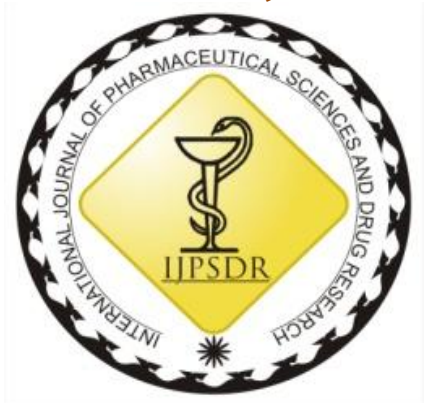

Research Article

ISSN: 0975-248X CODEN (USA): IJPSPP

(cc) BY-NC-SA

\title{
Synthesis, Spectral and Molecular Characterization of Some Novel 2, 5-Disubstituted-1, 3, 4-Oxadiazole Derivatives and Evaluation of in vivo Antitumour Activity against HT 29 Cell Line
}

\author{
Asish Bhaumik $^{1}$, M. Chinna Eswaraiah ${ }^{2}$, Raja Chakraborty $^{1}$ \\ ${ }^{1}$ Department of Pharmaceutical Sciences, Assam down town University, Gandhi Nagar, Panikhaiti, Guwahati- 781026, \\ Assam, India \\ 2Department of Pharmacognosy, Anurag Pharmacy College, Ananthagiri, Kodad-508206, Suryapet (Dist.), Telangana,
} India

Copyright ( $\odot 2018$ Asish Bhaumik et al. This is an open access article distributed under the terms of the Creative Commons AttributionNonCommercial-ShareAlike 4.0 International License which allows others to remix, tweak, and build upon the work non-commercially, as long as the author is credited and the new creations are licensed under the identical terms.

\begin{abstract}
Neoplasia is a type of abnormal and excessive growth of tissue. The growth of a neoplasia is uncoordinated with that of the normal surrounding tissue, and it persists growing abnormally, even if the original trigger is removed. This abnormal growth usually forms a mass. The main objective of the present research work was the synthesis, characterization and evaluation of in vivo antitumour activity of some novel 2, 5-disubstituted 1, 3, 4oxadiazole derivatives. The in vivo antitumour activity of synthesized compounds was evaluated by HT 29 cell line induced malignant ascites on mouse model. The apoptosis of HT 29 cells was evaluated by using Gimsa and H33342 stain and the apoptosis ratios were analysed by FCM using AnnexinV-FITC/PI staining. The present experimental data displayed that the mortality was less in all groups except in tumour control group and all the synthesized compounds AB1-AB8 (100 mg/ kg) significantly increased the PILS. While 5-FU increased the life span of $97.72 \%$, and the PILS of synthesized compounds were found to be $45.45 \%, 59.09 \%, 68.18 \%, 56.81 \%$, $38.63 \%, 84.09 \%, 77.27 \%$ and $90.90 \%$. So the Synthesized compounds AB1-AB8 at the dose of $100 \mathrm{mg} / \mathrm{kg}$ significantly improved the overall survival of all treated animals and 5-FU was not significantly differed from each other in improving the overall survival of HT-29 cells. The apoptosis ratios of synthesized compounds were found as followed: $\mathrm{AB} 1=26 \% ; \mathrm{AB} 2=37.6 \% ; \mathrm{AB} 3=43 \% ; \mathrm{AB} 4=29 \% ; \mathrm{AB} 5=24.1 \% ; \mathrm{AB} 6=59.2 \%$; $\mathrm{AB7}=48.2 \% ;$ and $\mathrm{AB} 8=63 \%$ respectively, while that of the Group-II (T. control) was $6.1 \%$. When compared with standard drug $5-$ FU: $66.2 \%$, it was indicated that compound AB8>AB6>AB7>AB3 were able to significantly induce HT-29 cells apoptosis.
\end{abstract}

Keywords: Neoplasia, antitumour, malignant ascites, HT 29, apoptosis, PILS.

DOI: 10.25004/IJPSDR.2018.100602

Int. J. Pharm. Sci. Drug Res. 2018; 10(6): 433-441

*Corresponding author: Mr. Asish Bhaumik

Address: Department of Pharmaceutical Sciences, Assam down town University, Gandhi Nagar, Panikhaiti, Guwahati- 781026, Assam, India E-mail $₫$ : bhaumik.asish@gmail.com

Relevant conflicts of interest/financial disclosures: The authors declare that the research was conducted in the absence of any commercial or financial relationships that could be construed as a potential conflict of interest.

Received: 20 July, 2018; Revised: 22 September, 2018; Accepted: 26 September, 2018; Published: 20 November, 2018 


\section{INTRODUCTION}

Oxadiazoles are a class of heterocyclic aromatic chemical compound of the azole family; with the molecular formula $\mathrm{C}_{2} \mathrm{H}_{2} \mathrm{~N}_{2} \mathrm{O}$. There are four isomers of oxadiazole depending on the position of nitrogen atom in the ring. ${ }^{11]} 1,3,4$-oxadiazole is a five membered heterocyclic aromatic compound containing two nitrogen atom at position three and four and one oxygen atom present at position one. 1, 3, 4 oxadiazole is thermally stable than other oxadiazoles, these oxadiazole are very important compound in medicinal chemistry due to their biological activities, during last few years. [2] 1,3, 4-oxadiazole is a liquid having boiling point $150^{\circ} \mathrm{C}$. 2, 5-disubstituted-1，3，4-oxadiazole derivatives are colorless substances. The lower alkyl derivatives are liquids which distil without decomposition. Replacement of an alkyl residue by an aryl radical considerably raises the melting and boiling points. Usually the asymmetrical 1, 3, 4-oxadiazole derivatives melt and boil at lower temperature than the symmetrical compounds. The solubility of oxadiazoles in water varies with the substituents present: 2, 5dimethyl-1, 3, 4-oxadiazole is miscible with water in all proportions whereas the solubility of 2, 5-diphenyl-1, 3, 4-oxadiazole in water is less. Electrophilic introduction of functional groups (for example nitro or sulphuric acid groups) into the nucleus is unusual. Electrophilic substitution occurs in aryl substituent. Halogenations are also difficult, but 2, 5-diaryl-1, 3, 4-oxadiazoles, afford complexes with halogens. A range of acylation and alkylation reactions of hydroxyl, thio and amino-1, 3, 4-oxadiazoles occur at the ring nitrogen. [3] Biologically active molecules containing oxadiazole moiety possessed a wide range of pharmacological activities such as antimicrobial, anticancer, anticonvulsant, anti-inflammatory and antiviral agents ${ }^{[4]}$, antifungal ${ }^{[5]}$, antimycobacterial ${ }^{[6]}$ etc.

\section{MATERIALS AND METHODS}

\section{Chemicals}

The solvents and other chemicals which were used for the synthesis and purification of target compounds provided by institutional store and were of $L R$ and AR grade.

\section{Instrumentation}

The melting points of the synthesized compounds were determined by open capillary tube method. The IR spectra of the synthesized compounds were recorded on ABB Bomen FT-IR spectrometer MB 104 IR spectra recorded with potassium bromide pellets. The ${ }^{1} \mathrm{H}-\mathrm{NMR}$ spectra of synthesized compounds were recorded on instrument BRUKER NMR spectrometer in DMSO. The Mass spectra of synthesized compounds were recorded JEOL GCmate. TLC method was used to determine the progress of the reaction. TLC plates are Pre-coated Silica gel (HF254-200 mesh) aluminium plates using ethyl acetate: n-hexane are used as solvent and visualized under UV- chamber. The IR, ${ }^{1} \mathrm{H}-\mathrm{NMR}$ and
MASS spectra were used to assign the structure of synthesized compounds.

Table 1: Oxadiazole<smiles>c1conn1</smiles><smiles>c1ncon1</smiles>

1,2,3-oxadiazole

1,2,4-oxadiazole

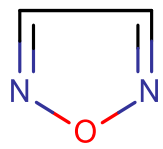

1,2,5-oxadiazole<smiles>c1nnco1</smiles>

1,3,4-oxadiazole

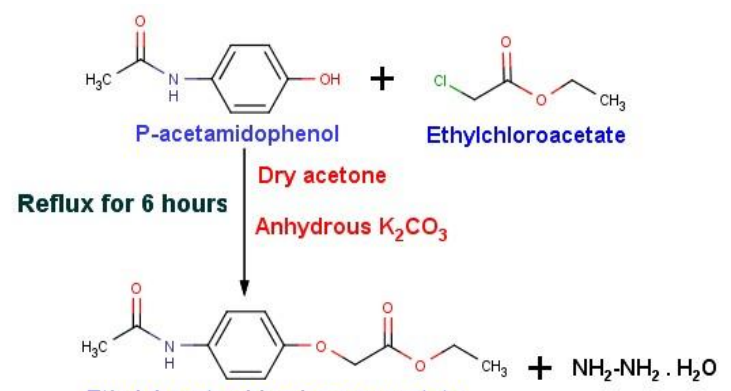

Hydrazine mono hydrate

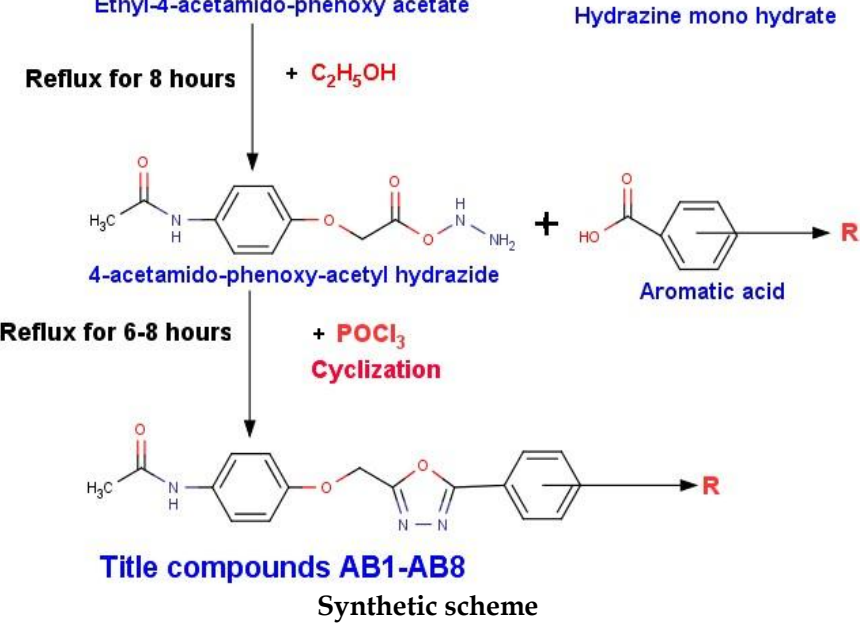

Steps involved in the synthesis of target compounds [7-9]

Step 1: Ethyl-4-acetamido phenoxy acetate

A mixture of p-acetamido phenol $(0.01 \mathrm{~mol})$ and ethyl chloroacetate $(0.01 \mathrm{~mol})$ was refluxed by using dry acetone in presence of anhydrous potassium carbonate $\left(\mathrm{K}_{2} \mathrm{CO}_{3}\right)$ for 6 hours. The reaction mixture was cooled and then poured in to crushed ice. The solid product obtained, these product was filtered, dried and recrystallized using ethanol.

Step 2: 4-Acetamido phenoxy acetyl hydrazide A mixture of ethyl-4-acetamido phenoxy acetate $(0.01$ $\mathrm{mol})$, hydrazine hydrate $(0.01 \mathrm{~mol})$ in ethanol $(15 \mathrm{ml})$ was refluxed for 5-8 hours. The reaction mixture was cooled and then poured in to crushed ice. The solid product was obtained; this product was filtered, dried and recrystallized from ethanol. 
Step 3: 2-(4-Acetamidophenoxy methyl) -5-aryl substituted - 1, 3, 4-oxadiazole

A mixture of 4-Acetamido phenoxy acetyl hydrazide $(0.01 \mathrm{~mol})$ and various aromatic acids $(0.01 \mathrm{~mol})$ in phosphorus oxychloride $(10 \mathrm{ml})$ was refluxed for $6-8$ hours. The completion of the reaction process was monitored by TLC plates. The contents were cooled and poured into the crushed ice and then neutralized the reaction mixture with sodium bicarbonate solution and the solid product was obtained; the product was filtered, dried and recrystallized from ethanol. [10]

Table 2: Physicochemical properties of synthesized compounds

\begin{tabular}{ccccccc}
\hline $\begin{array}{c}\text { S. } \\
\text { No. }\end{array}$ & $\begin{array}{c}\text { Compounds } \\
\text { code }\end{array}$ & M. F & M. Wt & $\begin{array}{c}\mathbf{R}_{\mathbf{f}} \\
\text { value }\end{array}$ & $\begin{array}{c}\mathbf{m} . \mathbf{p} \\
\left({ }^{\circ} \mathbf{C}\right)\end{array}$ & $\begin{array}{c}\text { Yield } \\
(\%)\end{array}$ \\
\hline 1. & $\mathrm{AB} 1$ & $\mathrm{C}_{17} \mathrm{H}_{16} \mathrm{~N}_{4} \mathrm{O}_{3}$ & 324.33 & 0.77 & 116 & 74.5 \\
2. & $\mathrm{AB} 2$ & $\mathrm{C}_{17} \mathrm{H}_{13} \mathrm{Cl}_{2} \mathrm{~N}_{3} \mathrm{O}_{3}$ & 378.20 & 0.74 & 180 & 69.9 \\
3. & $\mathrm{AB} 3$ & $\mathrm{C}_{17} \mathrm{H}_{14} \mathrm{FN}_{3} \mathrm{O}_{3}$ & 327.30 & 0.75 & 189 & 74 \\
4. & $\mathrm{AB} 4$ & $\mathrm{C}_{17} \mathrm{H}_{14} \mathrm{BrN}_{3} \mathrm{O}_{3}$ & 388.21 & 0.65 & 183 & 69 \\
5. & $\mathrm{AB} 5$ & $\mathrm{C}_{17} \mathrm{H}_{13} \mathrm{BrN}_{4} \mathrm{O}_{5}$ & 433.21 & 0.64 & 166 & 60 \\
6. & $\mathrm{AB} 6$ & $\mathrm{C}_{17} \mathrm{H}_{14} \mathrm{~N}_{4} \mathrm{O}_{5}$ & 354.31 & 0.72 & 171 & 64 \\
7. & $\mathrm{AB7}$ & $\mathrm{C}_{17} \mathrm{H}_{13} \mathrm{~N}_{5} \mathrm{O}_{7}$ & 399.31 & 0.68 & 204 & 78 \\
8. & $\mathrm{AB} 8$ & $\mathrm{C}_{17} \mathrm{H}_{13} \mathrm{~N}_{5} \mathrm{O}_{8}$ & 415.31 & 0.72 & 215 & 68 \\
\hline
\end{tabular}

Spectral data of synthesized compounds Compound AB1

N-(4-\{[5-(4-aminophenyl)-1,3,4-oxadiazol-2-

yl]methoxy\}phenyl) acetamide. IR $(\mathrm{KBr}) \mathrm{v}\left(\mathrm{cm}^{-1}\right)$ : $3393.16 \mathrm{~cm}^{-1}$ (Ar-NH), $1633.67 \mathrm{~cm}^{-1}(\mathrm{C}=\mathrm{N}), 1575.88 \mathrm{~cm}^{-1}$ $(\mathrm{C}=\mathrm{C}), 1069.05 \mathrm{~cm}^{-1}$ (-C-O-C-), $3132.54 \mathrm{~cm}^{-1}(\mathrm{Ar}-\mathrm{CH})$, $1249.43 \mathrm{~cm}^{-1}\left(\mathrm{Ar}-\mathrm{NH}_{2}\right),{ }^{1} \mathrm{H}-\mathrm{NMR} \delta$ (ppm): 6.45-7.4 (s, 8H, $\mathrm{Ar}-\mathrm{H}), 5.17$ (s, 2H,-CH$), 4.1\left(\mathrm{~s}, 2 \mathrm{H},-\mathrm{NH}_{2}\right), 2.05$ (s,1H, $\left.\mathrm{CH}_{3}\right), 8.05$ (s, $\left.1 \mathrm{H},-\mathrm{NH}\right)$, Mass (m/e value) \% relative abundance: $324.12\left(\mathrm{M}^{+}\right)$(5.1), $310.87(4)$, 296.22 (8.25), 282.76 (2.2), 272.38(2.32), 262.6432 (7.3), 248.34 (11), 217.12 (15), 207.14 (7), 116.67 (18), 58.33(B).

\section{Compound AB2}

N-(4-\{[5-(2,4-dichlorophenyl)-1,3,4-oxadiazol-2-

yl]methoxy\}phenyl) acetamide.. IR $(\mathrm{KBr}) \quad \mathrm{v} \quad\left(\mathrm{cm}^{-1}\right)$ : $3381.92 \mathrm{~cm}^{-1}$ (Ar-NH), $1673.42 \mathrm{~cm}^{-1}(\mathrm{C}=\mathrm{N}), 1545.03 \mathrm{~cm}^{-1}$ $(\mathrm{C}=\mathrm{C}), \quad 1085.04 \mathrm{~cm}^{-1}$ (-C-O-C-), $687.47 \mathrm{~cm}^{-1} \quad(\mathrm{C}-\mathrm{Cl})$, $3115.62 \mathrm{~cm}^{-1}$ (Ar-CH), ${ }^{1} \mathrm{H}-\mathrm{NMR} \delta$ (ppm): 6.6-7.82(s, 8H, Ar-CH), 2.5 (s, 3H, - $\left.\mathrm{CH}_{3}\right), 8.03(\mathrm{~s}, 1 \mathrm{H},-\mathrm{NH}), 5.22(\mathrm{~s}, 2 \mathrm{H}$, $\mathrm{CH}_{2}$ ), Mass (m/e value) \% relative abundance: 377.03 $\left(\mathrm{M}^{+}\right)$(2.8), $333.16(1.5), 325.42$ (2.7), 286.43 (2.6), 183.26 (6), 160.62 (7), 140.65 (16), 115.64 (33), 95.53 (B).

\section{Compound AB3}

N-(4-\{[5-(4-flurophenyl)-1,3,4-oxadiazol-2-

yl]methoxy\}phenyl) acetamide. IR $(\mathrm{KBr}) \mathrm{v}\left(\mathrm{cm}^{-1}\right)$ : $3392.09 \mathrm{~cm}^{-1}$ (Ar-NH), $1617.53 \mathrm{~cm}^{-1}(\mathrm{C}=\mathrm{N}), 1528.16 \mathrm{~cm}^{-1}$ (C=C), $1093.52 \mathrm{~cm}^{-1}$ (-C-O-C-), $1371.78 \mathrm{~cm}^{-1}$ (C-F), $3114.61 \mathrm{~cm}^{-1}$ (Ar-CH), ${ }^{1} \mathrm{H}-\mathrm{NMR} \delta$ (ppm): 2.21 (s, 1H, $\left.\mathrm{CH}_{3}\right), 8.09$ (s, 1H, $\left.-\mathrm{NH}\right), 5.21\left(\mathrm{~s}, 1 \mathrm{H},-\mathrm{CH}_{2}\right), 6.7-8.01(\mathrm{~m}$, $8 \mathrm{H}, \mathrm{Ar}-\mathrm{CH})$, Mass (m/e value) \% relative abundance: $327.10\left(\mathrm{M}^{+}\right)$(6.3), 310.37 (2.3), 299.57 (3), 282.87 (3.9), 266.22 (5), 249.61 (1.2), 232.72 (4), 104.86 (8.1), 75.50 (B).

\section{Compound AB4}

N-(4-\{[5-(2-bromophenyl)-1,3,4-oxadiazol-2-

yl]methoxy\}phenyl) acetamide. IR $(\mathrm{KBr}) \mathrm{v}\left(\mathrm{cm}^{-1}\right)$ : $3286.82 \mathrm{~cm}^{-1}$ (Ar-NH), $1617.53 \mathrm{~cm}^{-1}(\mathrm{C}=\mathrm{N}), 1528.16 \mathrm{~cm}^{-1}$ (C=C), $1093.52 \mathrm{~cm}^{-1}$ (-C-O-C-), $687.47 \mathrm{~cm}^{-1}$ (C-Br),
$3114.61 \mathrm{~cm}^{-1}$ (Ar-CH), ${ }^{1} \mathrm{H}-\mathrm{NMR} \delta(\mathrm{ppm}): 2.21$ (s, 1H, $\left.\mathrm{CH}_{3}\right), 8.09(\mathrm{~s}, 1 \mathrm{H},-\mathrm{NH}), 5.21\left(\mathrm{~s}, 1 \mathrm{H},-\mathrm{CH}_{2}\right), 6.7-8.01(\mathrm{~m}$, $8 \mathrm{H}, \mathrm{Ar}-\mathrm{CH})$, Mass (m/e value) \% relative abundance: 387.02( $\left.\mathrm{M}^{+}\right)$(6.3), 310.37 (2.3), 299.57 (3), 282.87 (3.9), 266.22 (5), 249.61 (1.2), 232.72 (4), 104.86 (8.1), 75.60 (B).

Compound AB5

N-(4-\{[5-(2-bromo,4-nitrophenyl)-1,3,4-oxadiazol-2-

yl]methoxy\}phenyl) acetamide. IR (KBr) v $\left(\mathrm{cm}^{-1}\right)$ : $3381.95 \mathrm{~cm}^{-1}$ (Ar-NH), $1684.44 \mathrm{~cm}^{-1}(\mathrm{C}=\mathrm{N}), 1586.2 \mathrm{~cm}^{-1}$ $(\mathrm{C}=\mathrm{C}), \quad 1064.25 \mathrm{~cm}^{-1}$ (-C-O-C-), $1365.57 \mathrm{~cm}^{-1}(\mathrm{~N}=\mathrm{O})$, $619.89 \mathrm{~cm}^{-1}$ (C-Br), $3130.43 \mathrm{~cm}^{-1}$ (Ar-CH), ${ }^{1} \mathrm{H}-\mathrm{NMR} \delta$ (ppm): 6.74-8.36(m, 7H, Ar-CH) ,5.31(s, $\left.2 \mathrm{H},-\mathrm{CH}_{2}\right), 2.31$ (s, $\left.1 \mathrm{H},-\mathrm{CH}_{3}\right), 8.16(\mathrm{~s}, 1 \mathrm{H},-\mathrm{NH})$, Mass (m/e value) \% relative abundance: $432.00\left(\mathrm{M}^{+}\right)(4), 388.71(8.1), 362.27$ (4.2), 233.28 (5), 217.31 (8.9), 182.52 (5), 96.79 (7), 78.82(B).

\section{Compound AB6}

N-(4-\{[5-(4-nitrophenyl)-1,3,4-oxadiazol-2-

yl]methoxy\}phenyl)acetamide. IR $(\mathrm{KBr}) \quad \mathrm{V} \quad\left(\mathrm{cm}^{-1}\right)$ : $3382.43 \mathrm{~cm}^{-1}$ (Ar-NH), $1703.01 \mathrm{~cm}^{-1}(\mathrm{C}=\mathrm{N}), 1592.32 \mathrm{~cm}^{-1}$ $(\mathrm{C}=\mathrm{C}), 1088.54 \mathrm{~cm}^{-1}$ (-C-O-C-), $1378.11 \mathrm{~cm}^{-1} \quad(\mathrm{~N}=\mathrm{O})$, $3112.69 \mathrm{~cm}^{-1}$ (Ar-CH), ${ }^{1} \mathrm{H}-\mathrm{NMR} \delta$ (ppm): 6.41-7.8(m, $8 \mathrm{H}$, Ar-CH), 2.42 (s, 3H, $\left.-\mathrm{CH}_{3}\right), 8.13(\mathrm{~s}, 1 \mathrm{H},-\mathrm{NH}), 5.21(\mathrm{~s}, 2 \mathrm{H}$, $\mathrm{CH}_{2}$ ), Mass (m/e value) \% relative abundance: 354.09 $\left(\mathrm{M}^{+}\right)$(3.8), 335.16 (4.8), 302.39 (3.1), 287.43 (3.7), 249.58 (7.1), 226.00 (5.8), 204.96 (6.7), 127.56 (13.1), 103.69 (9), 89.93 (B).

\section{Compound AB7}

N-(4-\{[5-(3,5-dinitrophenyl)-1,3,4-oxadiazol-2-

yl]methoxy\}phenyl) acetamide. IR $(\mathrm{KBr}) \mathrm{v}\left(\mathrm{cm}^{-1}\right)$ : $3382.02 \mathrm{~cm}^{-1}(\mathrm{Ar}-\mathrm{NH}), 1677.79 \mathrm{~cm}^{-1}(\mathrm{C}=\mathrm{N}), 1530.6 \mathrm{~cm}^{-1}$ $(\mathrm{C}=\mathrm{C}), 1089.68 \mathrm{~cm}^{-1}$ (-C-O-C-), $1372.45 \mathrm{~cm}^{-1} \quad(\mathrm{~N}=\mathrm{O})$, 1523.12 asym $\mathrm{cm}^{-1}(\mathrm{~N}=\mathrm{O}), 3117.5 \mathrm{~cm}^{-1}(\mathrm{Ar}-\mathrm{CH}),{ }^{1} \mathrm{H}-$ NMR $\delta$ (ppm): 6.83-8.42(m, 8H, Ar-CH), 5.35(s, 2H,$\left.\mathrm{CH}_{2}\right), 2.07$ (s, 1H,- $\left.\mathrm{CH}_{3}\right), 8.24(\mathrm{~s}, 1 \mathrm{H},-\mathrm{NH})$, Mass (m/e value) \% relative abundance: $399.08\left(\mathrm{M}^{+}\right)(5), 388.76$ (13), 380.25 (8), 261.63 (8), 182.52 (5), 167.62 (17), 156.56 (19), 81.97(B).

\section{Compound AB8}

N-(4-\{[5-(2-hydroxy-3,5-dinitrophenyl)-1,3,4-oxadiazol2-yl]methoxy\}phenyl) acetamide. IR $(\mathrm{KBr}) \mathrm{v}\left(\mathrm{cm}^{-1}\right)$ : $3118.84 \mathrm{~cm}^{-1}$ (Ar-NH), $1654.42 \mathrm{~cm}^{-1}(\mathrm{C}=\mathrm{N}), 1541.89 . \mathrm{cm}^{-1}$ $(\mathrm{C}=\mathrm{C}), \quad 1368.45 \mathrm{~cm}-1 \quad(\mathrm{~N}=\mathrm{O}), \quad 1528.45$ asym. $\mathrm{cm}-1$ $(\mathrm{N}=\mathrm{O}), 1090.01 \mathrm{~cm}^{-1}$ (-C-O-C-), $3118.84 \mathrm{~cm}^{-1}$ (Ar-CH), $3382.83 \mathrm{~cm}^{-1}(\mathrm{Ar}-\mathrm{OH}),{ }^{1} \mathrm{H}-\mathrm{NMR} \delta$ (ppm): 6.7-7.6(s, 6H, Ar-CH), 2.11 (s, H, - $\left.\mathrm{CH}_{3}\right), 8.00(\mathrm{~s}, 1 \mathrm{H},-\mathrm{NH}), 5.12(\mathrm{~s}, 1 \mathrm{H}$, $\mathrm{CH}_{2}$ ), Mass (m/e value) \% relative abundance: 415.07(M) (11.1), 318.68 (16), 292.76 (7), 276.89 (20), 249.99 (8.2), 236.0277 (28.1), 203.2266 (76), 182.2587 (8), 134.4966 (32), 116.55 (B).

\section{Experimental Pharmacology}

Requirements

Carboxymethyl cellulose sodium (CMC) was provided by institutional store. The standard drug 5-FU was purchased from local retail shop Apollo pharmacy, Jyothinagar, OMR road, Chennai. Female Swiss albino mice (20-30 g) were obtained from the central animal house of C. L. Baid Metha College of pharmacy, Jyothinagar, OMR, Chennai and they were maintained 
under standard laboratory conditions throughout the study. The study protocol was approved by the
Institutional Animal Ethics Committee

(IAEC) reference number: IAEC/XXIX/10/2017.

Table 3A: Molecular properties of synthesized compounds

\begin{tabular}{|c|c|c|c|c|c|c|c|c|}
\hline \multirow{2}{*}{$\begin{array}{l}\text { Compound } \\
\text { s codes }\end{array}$} & \multicolumn{4}{|c|}{ Polarisability } & \multicolumn{4}{|c|}{ Conformation (K.cal/mol) } \\
\hline & Molecular & a $(X X)$ & a (YY) & a $(\mathrm{ZZ})$ & Conf.1 & Conf.2 & Conf.3 & Conf.4 \\
\hline AB1 & 35.00 & 19.00 & 30.00 & 55.2 & 55.94 & 56.59 & 58.16 & 58.35 \\
\hline AB2 & 37.89 & 21.96 & 33.05 & 58.66 & 59.01 & 69.11 & 60.66 & 60.72 \\
\hline AB3 & 33.54 & 18.46 & 29.65 & 52.52 & 55.29 & 55.42 & 55.85 & 38.13 \\
\hline $\mathrm{AB} 4$ & 36.32 & 51.74 & 32.75 & 24.47 & 57.31 & 57.62 & 58.25 & 59.42 \\
\hline AB5 & 38.96 & 56.70 & 35.70 & 24.49 & 64.29 & 64.51 & 65.36 & 66.64 \\
\hline AB6 & 35.90 & 32.78 & 20.88 & 84.03 & 62.01 & 62.63 & 63.65 & 63.79 \\
\hline AB7 & 38.32 & 35.03 & 23.00 & 56.14 & 69.64 & 69.92 & 70.56 & 71.87 \\
\hline AB8 & 38.71 & 36.06 & 25.11 & 54.95 & 73.62 & 73.94 & 75.98 & 76.27 \\
\hline
\end{tabular}

Conf: Conformer

Table 3B: Molecular geometry of synthesized compounds

\begin{tabular}{|c|c|c|c|c|c|c|c|c|c|}
\hline \multirow{2}{*}{$\begin{array}{c}\text { Compounds } \\
\text { codes }\end{array}$} & \multicolumn{9}{|c|}{ Geometry } \\
\hline & $\mathrm{DE}$ & MMFF94 & Min.pa & Max.pa & Min.pr & Max.pr & Vdw.vol & Lp.max.a & Lp.min. a \\
\hline AB1 & 57.23 & 99.59 & 40.44 & 103.65 & 4.17 & 10.08 & 280.38 & 5.55 & 19.92 \\
\hline AB2 & 59.01 & 98.71 & 35.35 & 109.22 & 4.46 & 10.74 & 297.21 & 6.67 & 21.4 \\
\hline AB3 & 55.42 & 105.21 & 34.19 & 102.79 & 4.03 & 10.48 & 274.33 & 5.69 & 20.77 \\
\hline AB4 & 57.31 & 110.43 & 34.34 & 105.77 & 4.17 & 10.00 & 287.82 & 5.70 & 19.91 \\
\hline AB5 & 64.51 & 148.45 & 34.38 & 112.98 & 4.18 & 10.79 & 310.7 & 5.75 & 21.57 \\
\hline AB6 & 62.53 & 156.94 & 37.72 & 107.67 & 4.62 & 10.66 & 292.24 & 7.19 & 21.16 \\
\hline AB7 & 69.64 & 172.16 & 41.05 & 115.79 & 4.94 & 10.61 & 315.35 & 6.14 & 21.12 \\
\hline AB8 & 73.62 & 186.39 & 42.39 & 118.30 & 4.97 & 10.58 & 324.00 & 6.18 & 21.10 \\
\hline
\end{tabular}

DE: Dreiding energy (kcal/mol); MMFF94 energy (kcal/mol); Min. Pa: Minimal projection area; Max.pa: Maximal projection area; Min.pr: Minimal projection radius; Max.pr: Maximal projection radius; LP.max.a: Length perpendicular to the max area; Lp. mina: Length perpendicular to the min; Van der Waals volume

Table 3C: Molecular properties of synthesized compounds

\begin{tabular}{|c|c|c|c|c|c|c|c|}
\hline \multirow{2}{*}{$\begin{array}{c}\text { Compounds } \\
\text { codes }\end{array}$} & \multirow{2}{*}{ PSA(2D) } & \multirow{2}{*}{ Vdw.SA(3D) } & \multicolumn{4}{|c|}{ H-bonding } & \multirow{2}{*}{ Refractivity } \\
\hline & & & Ds & Dc & As & Ac & \\
\hline AB1 & 103.27 & 445.73 & 03 & 02 & 06 & 05 & 102.05 \\
\hline AB2 & 77.25 & 461.95 & 01 & 01 & 05 & 04 & 106.96 \\
\hline AB3 & 77.25 & 437.77 & 01 & 01 & 05 & 04 & 97.57 \\
\hline AB4 & 77.25 & 449.66 & 01 & 01 & 05 & 04 & 104.97 \\
\hline AB5 & 123.07 & 489.50 & 01 & 01 & 09 & 06 & 112.30 \\
\hline AB6 & 123.07 & 470.58 & 01 & 01 & 09 & 06 & 104.68 \\
\hline AB7 & 168.89 & 510.43 & 01 & 01 & 13 & 08 & 112.00 \\
\hline AB8 & 189.12 & 519.03 & 02 & 02 & 14 & 09 & 113.98 \\
\hline
\end{tabular}

PSA(2D): Polar surface area; Vdw.SA(3D): Van der Waals surface area; Ds: Donor sites; Dc: Donor count; As: Acceptor sites; Ac: Acceptor count.

\section{Determination of median lethal doses $\left(\mathrm{LD}_{50}\right)$}

In the present study acute oral toxicity of the synthesized compounds were performed by acute toxic class method according to OECD guideline-423. [9]

Cell culturing

HT 29 human colorectal cancer cells were purchased from Amala Cancer Research Centre, Thrissur, and Kerala.

Study design [10]

An investigational study was designed to evaluate the in vivo antitumor activity of synthesized compounds (AB1-AB8) on mouse tumour models. Study was carried out with HT 29 cell line induced malignant ascites on mouse models. The dose of synthesized compounds $100 \mathrm{mg} / \mathrm{kg}$ were chosen based on the results of a toxicity study done previously. The animals were divided into eleven groups (each group contain 6 mice) as follows:

A. Group I: Normal Control Group [only the vehicle (1 $\mathrm{ml} / \mathrm{kg} /$ day of $1 \%$ CMC orally)]
B. Group II: T. Control (1\% CMC orally + HT $29=2 \times 10^{6}$ i.p.)

C. Group III: Standard (HT $29=2 \times 10^{6}$ i.p. +5 -FU 25 $\mathrm{mg} / \mathrm{ml}$ inj.)

D. Group IV-XI: AB1-AB8 (HT $29=2 \times 10^{6}$ i.p. +100 $\mathrm{mg} / \mathrm{kg}$ orally)

(Group IV: AB1, Group V: AB2, Group VI: AB3, Group VII: AB4, Group VIII: AB5, Group IX: AB6, Group X: AB7, Group XI: AB8)

Table 4: Designing of experiment [11]

\begin{tabular}{ccc}
\hline Days & Activity was carried out & $\begin{array}{c}\text { No. of mice / } \\
\text { group }\end{array}$ \\
\hline $\begin{array}{c}\text { Day 1 } \\
\text { Day 2 }\end{array}$ & $\begin{array}{c}\text { Collection of 0.3 ml of blood sample } \\
\text { Tumour cell injection, HT 29 }=2 \times 10^{6} \text { i.p. } \\
\text { Treatment of CMC }\end{array}$ & $\begin{array}{c}\text { Group-I-XI } \\
\text { Group-II-XI } \\
\text { Group-II }\end{array}$ \\
Day 3-12 & $\begin{array}{c}\text { Treatment of std. drug 5-FU } \\
\text { Treatment of Synthesized compounds } \\
\text { (AB1-AB8) }\end{array}$ & Group-IV -XI \\
$\begin{array}{c}\text { Day 15 } \\
\text { Day 16-35 } \\
\text { follow up }\end{array}$ & Collection of 0.3 ml of blood sample & Group-II-XI \\
\hline
\end{tabular}


Asish Bhaumik et al. / Synthesis, Spectral and Molecular Characterization of Some Novel 2, 5-Disubstituted.....

Table 5A: The assessment of haematological parameters

\begin{tabular}{|c|c|c|c|c|}
\hline Group & Treatment & $\begin{array}{c}\mathrm{Hb}(\mathrm{g} / \mathrm{dl}) \\
(\mathrm{MEAN} \pm \mathrm{SEM})\end{array}$ & $\begin{array}{c}\text { RBCs }\left(1 \times 10^{6} / \mathrm{mmm}^{3}\right) \\
(\text { MEAN } \pm \text { SEM) }\end{array}$ & $\begin{array}{c}\text { WBCs }\left(1 \times 10^{3} / \mathrm{mm}^{3}\right) \\
(\text { MEAN } \pm \text { SEM) }\end{array}$ \\
\hline I & N. Control & $13.62 \pm 0.213$ & $9.405 \pm 0.666$ & $6.032 \pm 0.007$ \\
\hline II & T. Control & $7.275 \pm 0.259$ & $6.19 \pm 0.096$ & $8.455 \pm 0.348$ \\
\hline III & $5-\mathrm{FU}^{* * *}$ & $12.7 \pm 0.204$ & $9.353 \pm 0.183$ & $6.17 \pm 0.001$ \\
\hline IV & AB1 & $7.825 \pm 0.301$ & $8.1 \pm 0.105$ & $6.44 \pm 0.024$ \\
\hline V & $\mathrm{AB} 2^{* *}$ & $10.35 \pm 0.246$ & $8.55 \pm 0.0108$ & $6.416 \pm 0.029$ \\
\hline VI & $\mathrm{AB}^{* *}$ & $10.65 \pm 0.184$ & $8.683 \pm 0.027$ & $6.294 \pm 0.024$ \\
\hline VII & $\mathrm{AB} 4$ & $8.05 \pm 0.225$ & $8.365 \pm 0.022$ & $6.725 \pm 0.195$ \\
\hline VIII & AB5 & $7.625 \pm 0.239$ & $8.45 \pm 0.167$ & $6.957 \pm 0.295$ \\
\hline IX & $\mathrm{AB} 6^{* * *}$ & $11.88 \pm 0.946$ & $8.843 \pm 0.028$ & $6.173 \pm 0.019$ \\
\hline $\mathrm{X}$ & $\mathrm{AB} 7^{* * *}$ & $11.53 \pm 0.149$ & $8.755 \pm 0.0202$ & $6.284 \pm 0.032$ \\
\hline XI & $\mathrm{AB} 8^{* * *}$ & $12.43 \pm 0.179$ & $8.955 \pm 0.055$ & $6.168 \pm 0.007$ \\
\hline
\end{tabular}

Table 5B: The assessment of haematological parameters

\begin{tabular}{ccccc}
\hline Group & Treatment & $\begin{array}{c}\text { Neutrophils (\%) } \\
\text { (MEAN } \pm \text { SEM) }\end{array}$ & $\begin{array}{c}\text { Lymphocytes (\%) } \\
\text { (MEAN } \pm \text { SEM) }\end{array}$ & $\begin{array}{c}\text { Platelets }\left(\mathbf{1} \times \mathbf{1 0}^{5} / \mathbf{m m}^{3}\right) \\
(\mathbf{M E A N} \pm \text { SEM) }\end{array}$ \\
\hline I & N. Control & $14.1 \pm 0.294$ & $13.33 \pm 0.125$ & $4.499 \pm 0.0009$ \\
II & T. Control & $86.24 \pm 0.745$ & $7.856 \pm 0.021$ & $12.85 \pm 0.248$ \\
III & $5-F U^{* * *}$ & $14.47 \pm 0.211$ & $12.14 \pm 0.016$ & $4.574 \pm 0.006$ \\
IV & AB1 & $15.83 \pm 0.058$ & $8.325 \pm 0.047$ & $5.431 \pm 0.0326$ \\
V & AB2 & $15.13 \pm 0.243$ & $10.1 \pm 0.238$ & $5.177 \pm 0.004$ \\
VI & AB3 & $14.72 \pm 0.072$ & $10.32 \pm 0.235$ & $5.017 \pm 0.028$ \\
VII & AB4 & $15.07 \pm 0.125$ & $8.52 \pm 0.033$ & $5.253 \pm 0.003$ \\
VIII & AB5 & $15.24 \pm 0.099$ & $8.538 \pm 0.209$ & $5.367 \pm 0.005$ \\
IX & AB6 & $11.25 \pm 0.159$ & $4.943 \pm 0.004$ \\
X & AB7** & $14.78 \pm 0.346$ & $10.87 \pm 0.141$ & $4.973 \pm 0.007$ \\
XI & AB8 & $14.82 \pm 0.32$ & $11.74 \pm 0.436$ & $4.704 \pm 0.002$ \\
\hline
\end{tabular}

Table 6: Comparison PILS (\%) in different treatment groups

\begin{tabular}{cccc}
\hline Group & Treatment & $\begin{array}{c}\text { MST(days) } \\
\text { (MEAN } \pm \text { SEM) }\end{array}$ & PILS (\%) \\
\hline II & T. Control & $4.4 \pm 0.04$ & $-----\leq----$ \\
III & 5-FU*** & $8.725 \pm 0.025$ & 97.72 \\
IV & AB1 & $6.4 \pm 0.04$ & 45.45 \\
V & AB2* & $7.05 \pm 0.064$ & 59.09 \\
VI & AB3* & $7.45 \pm 0.028$ & 68.18 \\
VII & AB4 & $6.8 \pm 0.04$ & 56.81 \\
VIII & AB5 & $6.125 \pm 0.025$ & 38.63 \\
IX & AB6** & $8.25 \pm 0.064$ & 84.09 \\
X & AB7** & $7.75 \pm 0.028$ & 77.27 \\
XI & AB8** & $8.475 \pm 0.047$ & 90.90 \\
\hline
\end{tabular}

$P<0.001=* * *$, highly significant. $P<0.01=* *$, moderate significant $P<0.05=*$, significant. $P>0.05=$ ns. Values are expressed as MEAN \pm SEM of 6 animals. The data were statistically analysed by ONE WAY

ANOVA followed by Tukey Kramer multiple comparison test.

Determination of the percentage increase in life span (PILS): It is calculated from the mean survival time (MST) values. [12] The MST for each group was calculated as: MST (days) = Total number of days survived by all animals in the group/Number of animals in the group. For each group, Percent increase of lifespan (\% ILS) was determined by the following formula: PILS $(\%)=[($ MST of treated group/MST o f control group) -1] $\times 100$. The haematological parameters of all surviving animals such as haemoglobin, RBC, WBC, neutrophils, lymphocytes and platelets were assessed for all.

Apoptosis analysis

Cardinal morphological features of apoptotic cells are determined by Gimsa staining technique [13], H33342 staining technique, annexin $\mathrm{V}$ staining ${ }^{[14-16]}$ by fluorescence microscopy.

\section{RESULTS AND DISCUSSION}

Assessment of haematological parameters

Effect on the haematological parameters

The present experimental data displayed that the mortality was less in all groups except in tumour control group. The haemoglobin and RBCs count were significantly lower in tumour control group compared to normal control group and significantly raise nearly to normal in all treatment groups when compared with control group. The WBC counts were significantly increased in tumour control and it came down to nearly normal range in all treatment groups. The neutrophils were increased and lymphocytes were decreased significantly in tumour control groups and significantly decreased neutrophils and increased lymphocytes in all treatment groups. The platelet count was significantly increased in tumour control (except Group-III to XI) group compared to normal group.

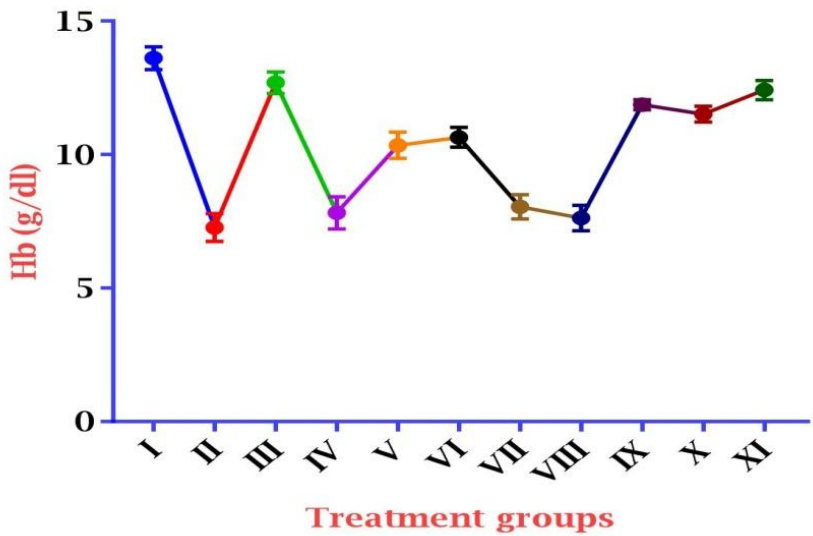

Fig. 1: Comparison of $\mathrm{Hb}$ level between different groups received HT29 tumour cells. 


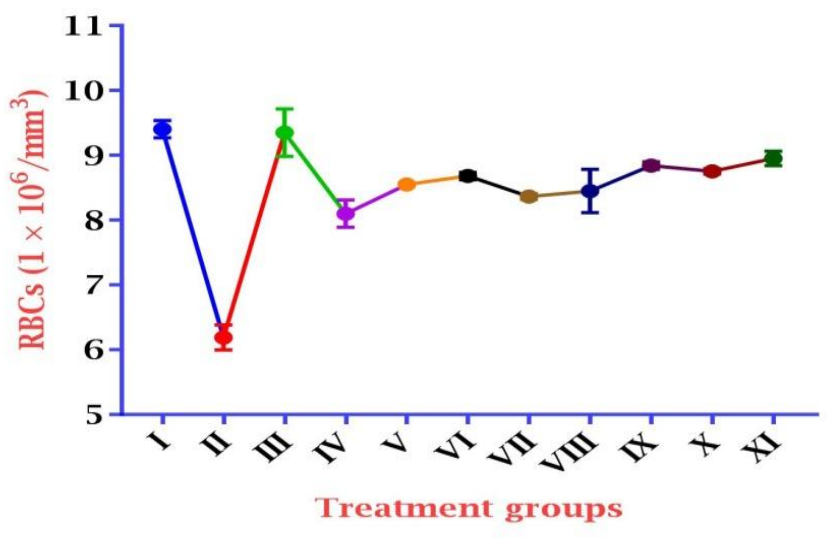

Fig. 2: Comparison of RBCs level between different groups received HT29 tumour cells.

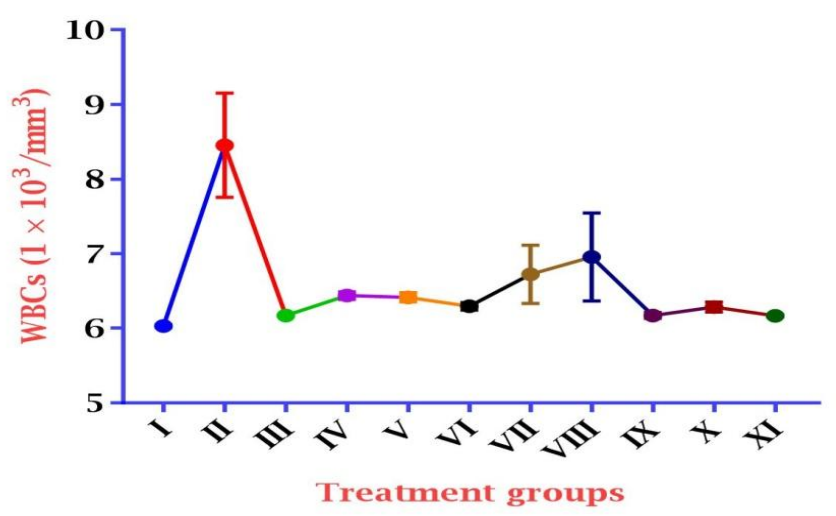

Fig. 3: Comparison of WBCs level between different groups received HT29 tumour cells.

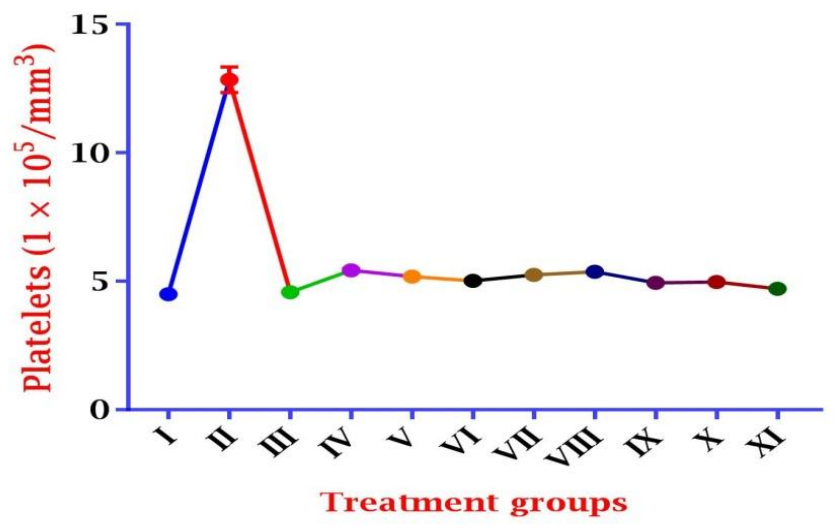

Fig. 4: Comparison of platelets count between different groups received HT29 tumour cells.

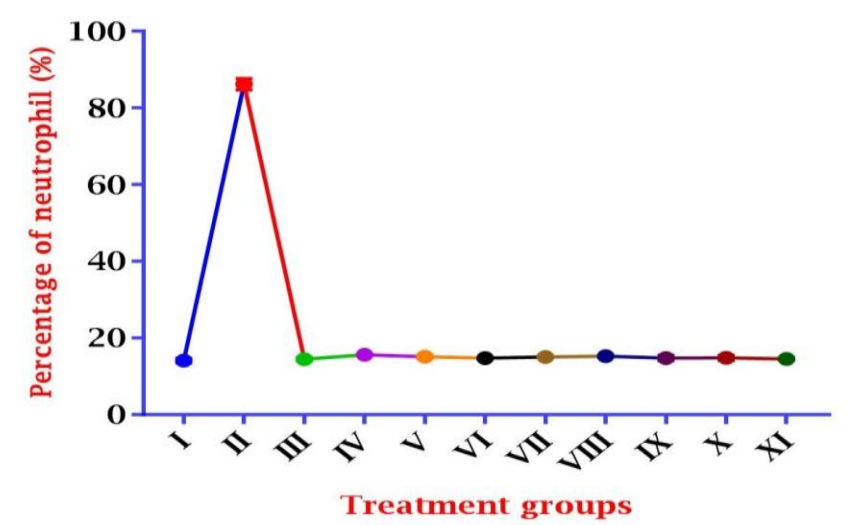

Fig. 5: Comparison of $\%$ of neutrophil between different groups received HT29 tumour cells.

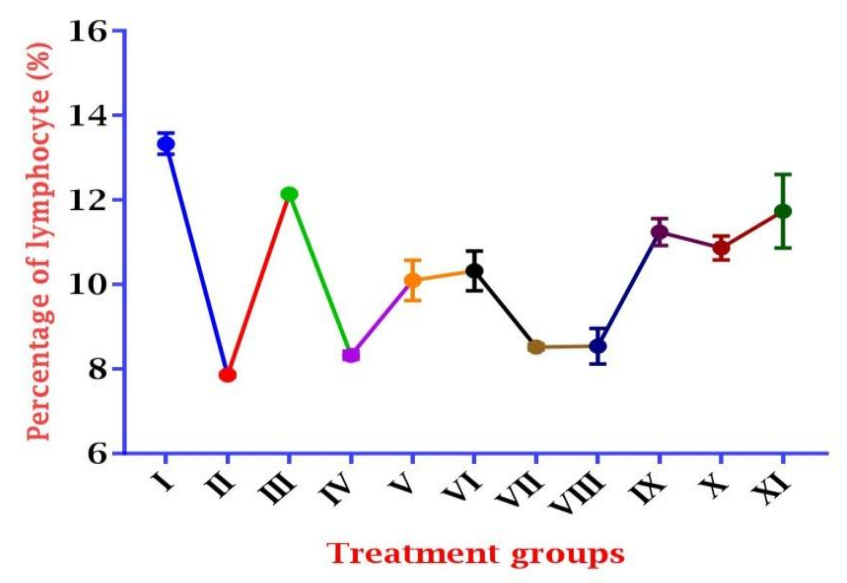

Fig. 6: Comparison of $\%$ of lymphocytes between different groups received $\mathrm{HT} 29$ tumour cells.

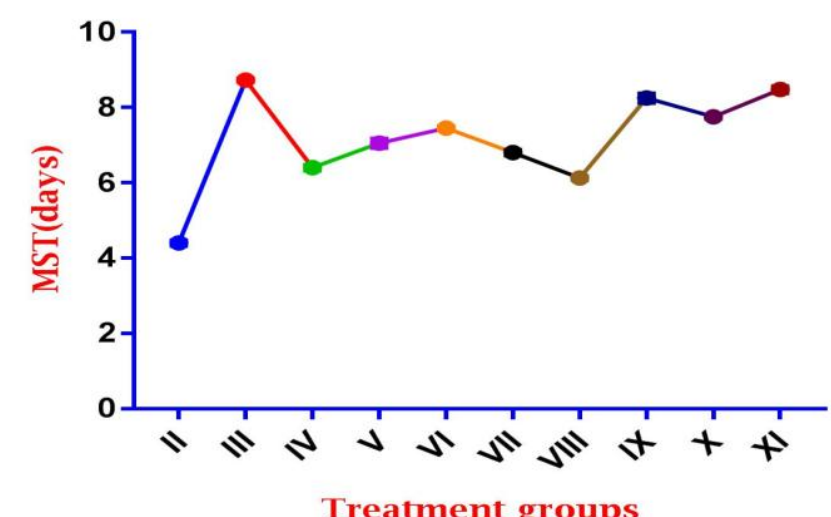

Fig. 7: Comparison of MST between different groups received HT29 tumour cells.

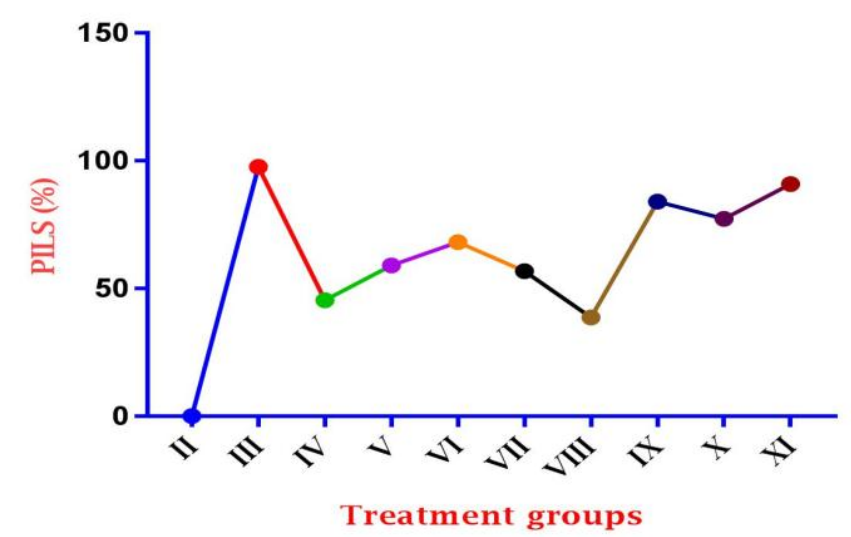

Fig. 8: Comparison of PILS between different groups received HT29 tumour cells.

Effect on the survival

All the synthesized compounds AB1-AB8 (100 mg/kg) significantly increased the PILS. While 5-FU increased the life span of $97.72 \%$, and the PILS of synthesized compounds were found to be $45.45 \%, 59.09 \%, 68.18 \%$, $56.81 \%, 38.63 \%, 84.09 \%, 77.27 \%$ and $90.90 \%$. So the Synthesized compounds AB1-AB8 at the dose of 100 $\mathrm{mg} / \mathrm{kg}$ significantly improved the overall survival of all treated animals and 5-FU was not significantly differed from each other in improving the overall survival of HT-29 cells. 

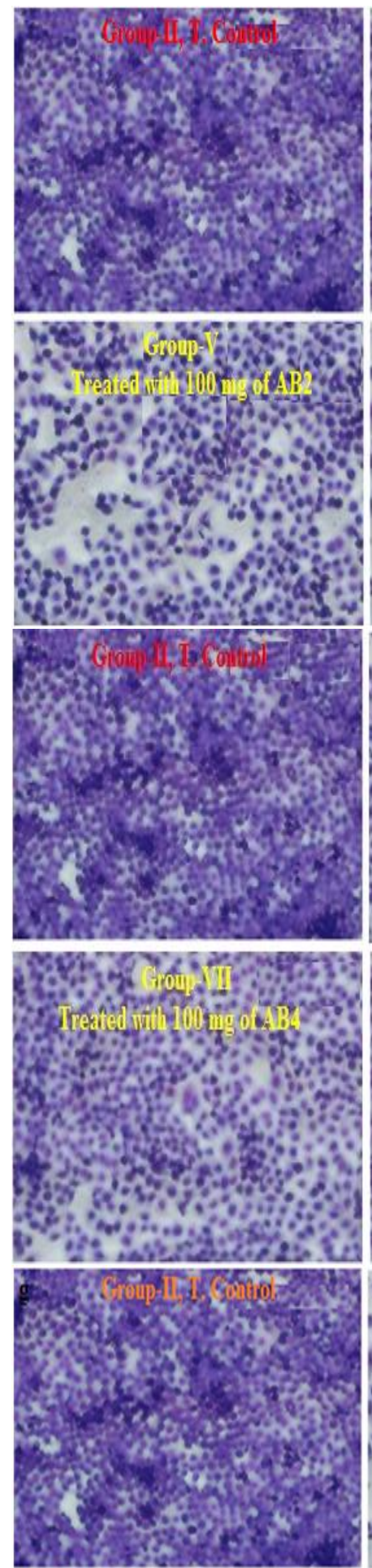

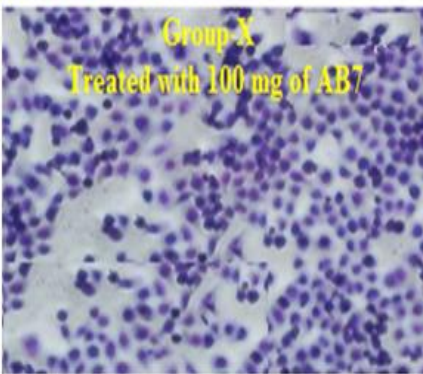
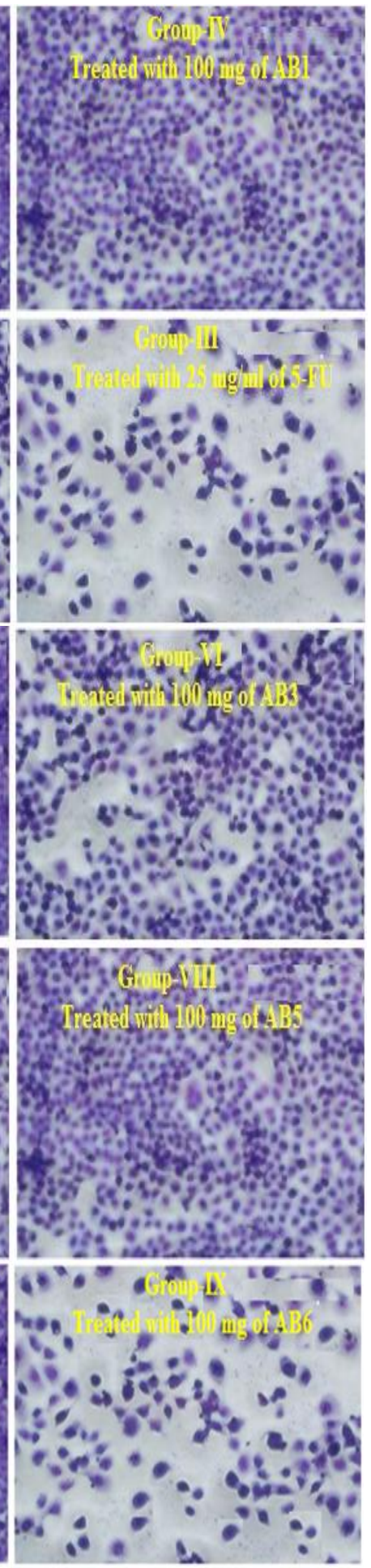

Fig. 9: Morphological changes observation of HT-29 cells by Giemsa staining (X200): Group-II: Tumour control; Group-III: Treated with standard drug and Group-IV-XI: Treated with $100 \mathrm{mg}$ of synthesized compounds AB1-AB8. The cell morphology was observed and photographed under inverted phase-contrast microscope after Giemsa staining.
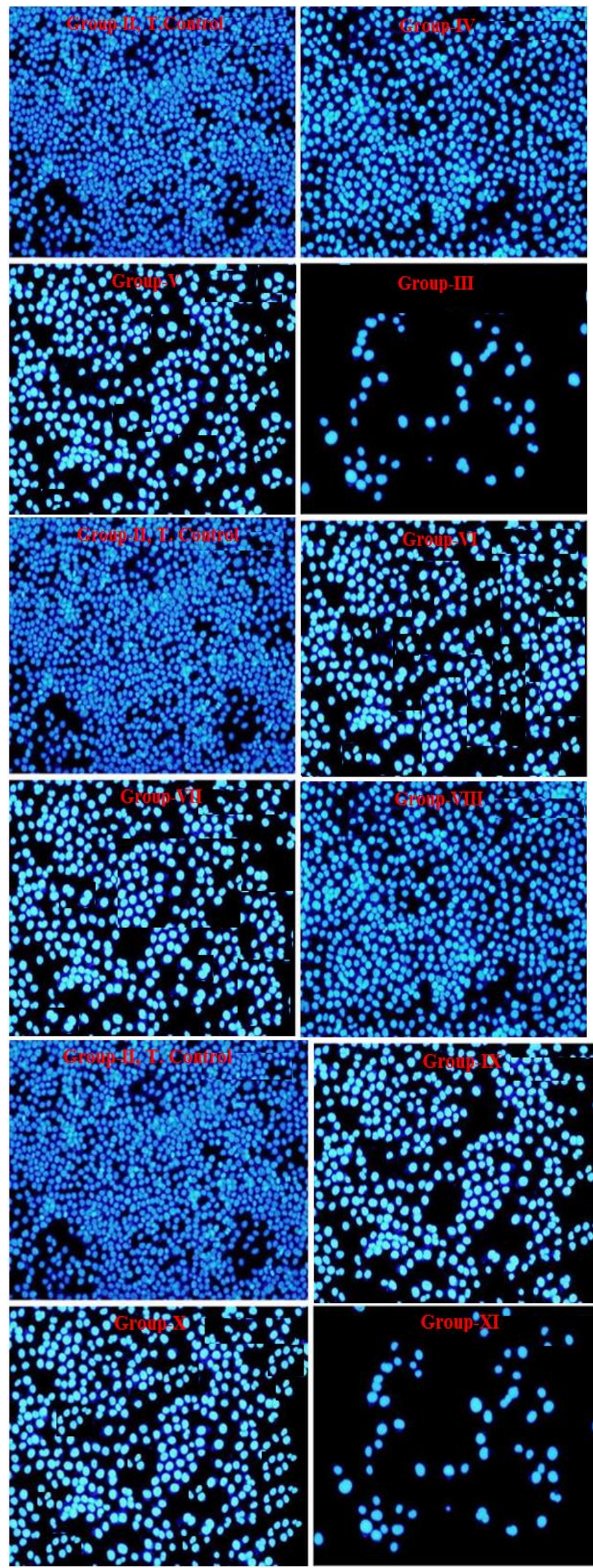

Fig. 10: Morphological changes observation of HT-29 cells by H33342 staining (X200): Group-II: T. Control; Group-III: Treated with standard drug 5-FU and Group-IV to Group-XI: Animals are treated with synthesized compounds (AB1-AB8). 

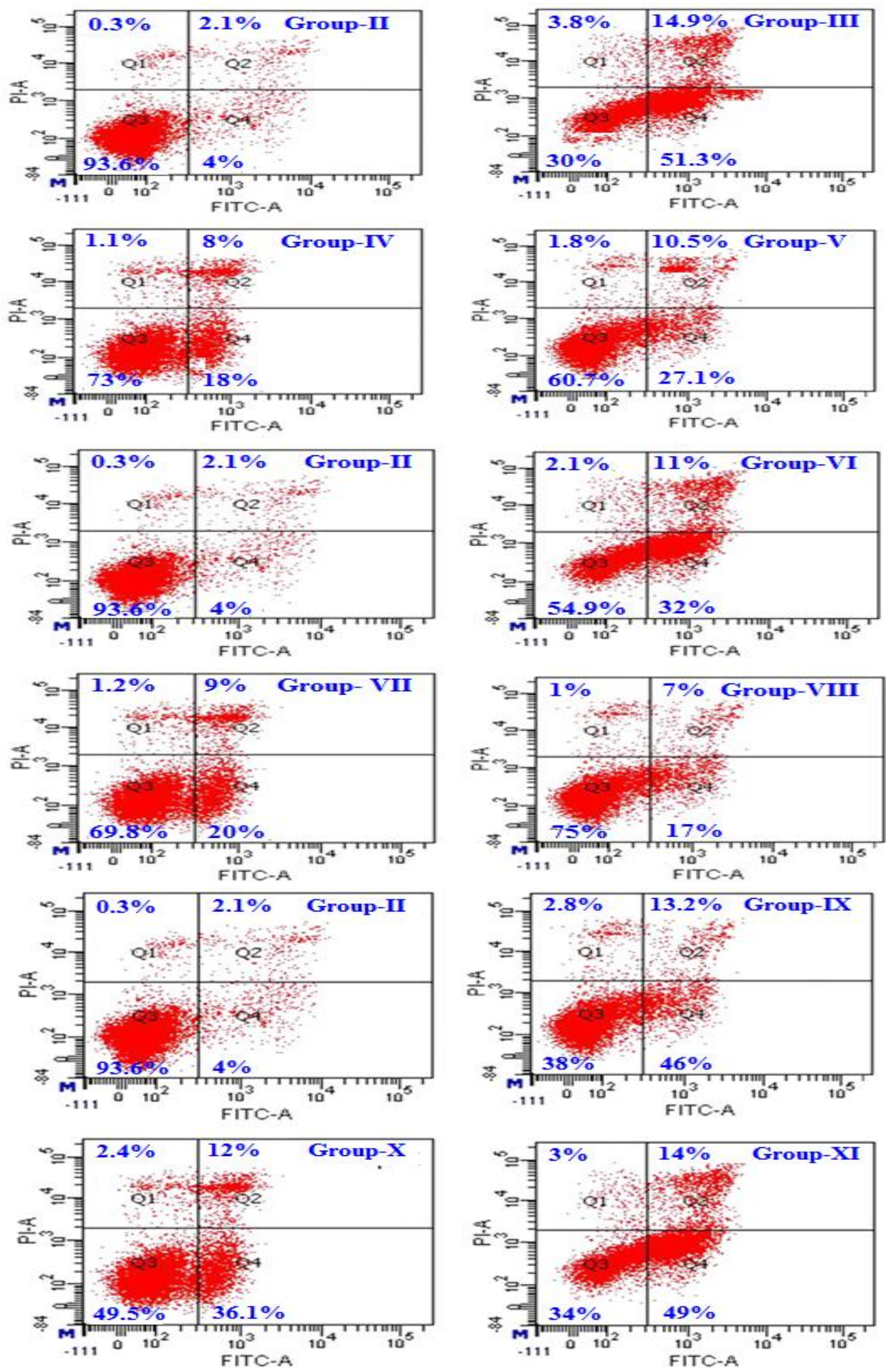

Fig. 11: Apoptosis analysis by FCM using AnnexinV-FITC/PI staining on the HT-29 cells treated by synthesized compounds; Group-II: Tumour control without compound and Group-III: Treated with standard drug 5-FU and Group-IV-XI: Treated with 100 mg of synthesized compounds: AB1-AB8.

Apoptosis analysis

\section{Giemsa staining}

To confirm whether the apoptotic morphological changes could be associated with the synthesized compounds, HT-29 cells were stained with Giemsa. The morphology changes were observed and photographed under inverted phase-contrast microscope at a magnification of 200X. With the treatment of tests compounds, the process of cell loss, nuclei lysis, chromatin condensation and cytoplasmic shrinkage were aggravated.

H33342 staining on HT-29 cells

In the group-II i, e tumour control group, HT 29 cell nuclei displayed a normal and complete blue 
appearance. By contrast, in HT 29 cells received groups i, e group-IV-XI which were treated with standard drug 5-FU (group-III), synthesized compounds (AB1-AB8), the cells displayed enhanced fragmentation or pyknosis of the nuclei, which were typical changes associated with cellular apoptosis. When compared with standard drug 5-FU, nuclear pyknosis and fragmentation in HT 29 cells were significantly increased by treatment with compounds $\mathrm{AB} 8<\mathrm{AB} 6<\mathrm{AB} 7<\mathrm{AB} 3<\mathrm{AB} 2$ and $\mathrm{AB} 4$ among the eight synthesized compounds.

\section{Annexin V-FITC/propidium iodide (PI) assay}

The effects of synthesized compounds on apoptosis in HT-29 cells were further determined by flow cytometric analysis. Cells were stained with both annexin V-FITC and PI. The flow cytometry observed four quadrant images: the Q1 area represented necrotic cells, the Q2 area represented late apoptotic cells, the Q3 area represented intact cells and the Q4 area represented the early apoptotic cells. The apoptosis ratios of different treatment groups were found as followed: Group$\mathrm{IV}=26 \%$ ； Group-V=37.6\%； Group-VI=43\%； Group$\mathrm{VII}=29 \%$; Group-VIII=24.1\%; Group-IX=59.2\%; Group$X=48.2 \%$; and $X I=63 \%$ respectively, while that of the Group-II (T. control) was 6.1\%. When compared with Group-III (standard drug 5-FU: 66.2\%), it was indicated that compound $\mathrm{AB} 8>\mathrm{AB} 6>\mathrm{AB} 7>\mathrm{AB} 3$ were able to significantly induce HT-29 cells apoptosis.

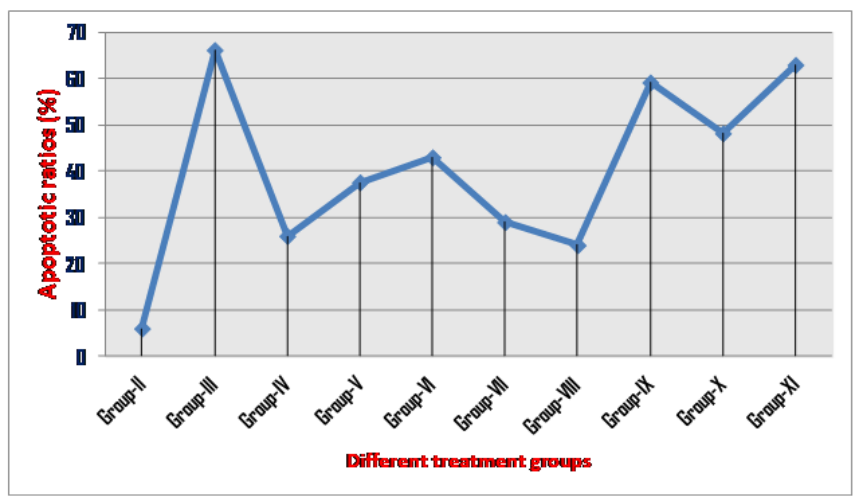

Fig. 12: Percentage of HT 29 cells death in different treatment groups.

In vivo experimental data showed that all the synthesized compounds possessed a mild to good antitumor activity against human colon cancer cell line HT29. Here it was found that among the all eight synthesized compounds the following compounds executed excellent antitumor activity against HT 29 cell line: AB2, AB3, AB6, AB7 and AB8 and apoptosis of HT 29 cell lines caused by synthesized compounds was evaluated by Giemsa staining and H33342 staining and cells death of HT 29 was further confirmed by Annexin V-FITC/propidium iodide (PI) assay.

\section{REFERENCES}

1. Gupta RR, Kumar M, Gupta V. Heterocyclic Chemistry. Volume II: Five membered Heterocycles India, 1st Edn, Springer, 2005, pp. 525-546.

2. Hetzheim A, Möckel K. Recent advances in 1, 3, 4-oxadiazole chemistry. Adv. Heterocycl. Chem. 1967; 7: 183-224.

3. Kharb R, Sharma PC, Bhandari A, Yar MS. Synthesis, spectral characterization and anthelmintic evaluation of some novel imidazole bearing triazole derivatives. Der Pharmacia Lettre 2012; 4(2): 652-657.

4. Santagati M, Modica M, Santagati A, Russo F, Caruso A, Cutuli V, Di Pietro E, Amico-Roxas M. Synthesis and pharmacological properties of benzothiazole,1,3,4-oxadiazole and 1,3,4-thiadiazole derivatives. Pharmazie. 1994; 49(12): $880-884$

5. Li Y, Liu J, Zhang H, Yang X, Liu Z. Stereoselective synthesis and fungicidal activities of (E)-alpha-(methoxyimino)benzeneacetate derivatives containing 1,3,4-oxadiazole ring Bioorganic \& Medicinal Chemistry Letters. 2006; 16(8):22782282.

6. Kucukguzel SG, Oruc EE, Rollas S, Sahin F, Ozbek A. Synthesis, characterisation and biological activity of novel 4thiazolidinones, 1,3,4-oxadiazoles and some related compounds. Eur. J. Med. Chem. 2002; 37(3): 197-206.

7. Ilango K, Valentina P, Umarani N, Kumar T. Synthesis and characterization of 2,5-disubstituted-1,3,4-oxadiazoles as potential inflammatory agents. J Young Pharm. 2009; 1(1):7276.

8. Jakubkiene V, Burbuliene MM, Mekuskiene G, Udrenaite E, Gaidelis P, Vainilavicius P. Synthesis and anti-inflammatory activity of 5-(6-methyl-2-substituted 4pyrimidinyloxymethyl)-1,3,4-oxadiazole-2-thiones and their 3-morpholinomethyl derivatives. Farmaco. 2003; 58:323-328.

9. OECD Guidelines for the Testing of Chemicals, Volume 1, Number 4, September 2006, pp. 1-14(14).

10. Boik J. Natural Compounds in Cancer Therapy. Oregon Medical Press, Minnesota, USA, 2001, pp. 25.

11. Thummar VR, Parasuraman S, Basu D, Raveendrana R. Evaluation of in vivo antitumor activity of cleistanthin B in Swiss albino mice. J Tradit Complement Med. 2016 Oct; 6(4): 383-388.

12. Raihan MO, Tareq SM, Brishti A, Alam MK, Haque A, Ali MS. Evaluation of antitumor activity of Leea indica (Burm.f.) Merr. Extract against Ehrlich ascites carcinoma (EAC) bearing Mice. Am J Biomed Sci. 2012; 4(2):143-152.

13. Chih HW, Chiu HF, Tang KS, Chang FR, Wu YC. Bullatacin a potent antitumor annonaceous acetogenin, inhibits proliferation of human by apoptosis induction. Life Sci. 2001; 69(11):1321-1331.

14. Lallemand B, Gelbcke M, Dubois J, Prévost M, Jabin I, Kiss R. Structure-activity relationship analyses of glycyrrhetinic acid derivatives as anticancer agents. Mini Rev Med Chem. 2011; 11(10):881-887.

15. Wang P, She G, Yang Y, Li Q, Zhang H, Liu J, Cao Y, Xu X, Lei $H$. Synthesis and biological evaluation of new ligustrazine derivatives as antitumour agents. Molecules 2012; 17(5):4972-4985.

16. Gupta SK, editor. Drug Screening Methods (Preclinical evaluation of New Drugs) 2nd ed. New Delhi: Jaypee Brothers Medical Publishers, 2010, pp. 214.

HOW TO CITE THIS ARTICLE: Bhaumik A, Chinna Eswaraiah M, Chakraborty R. Synthesis, Spectral and Molecular Characterization of Some Novel 2, 5-Disubstituted-1, 3, 4-Oxadiazole Derivatives and Evaluation of in vivo Antitumour Activity against HT 29 Cell Line. Int. J. Pharm. Sci. Drug Res. 2018; 10(6): 433-441. DOI: 10.25004/IJPSDR.2018.100602 